\title{
Interactive comment on "Regionalising rainfall-runoff modelling for predicting daily runoff in continental Australia" by H. Li and Y. Zhang
}

\author{
H. Li and Y. Zhang \\ yongqiang.zhang@csiro.au
}

Received and published: 6 December 2016

First, we would like to thank the critical review from this anonymous referee, and thank the HESS editorial office to provide us an opportunity to clarify the concerns and address the queries. We have copied the comments one by one and each of them is followed by response (Re:).

I. General comments The overall impression of this paper is that: not good structured, not clear on the purpose and novelty of this study, no sufficient support for the conclusion and poor language (not interesting to read and the text very hard to follow)..

Re: This comment is not professional at all. It seems that it can apply to any manuscript. It is indeed astonished. We believe that this paper has enough novelty deserving to be published in HESS. The novelty of this study includes that (1) 
it use $600+$ catchments for continental regionalisation study, which is indeed one of few studies focusing on such a large scale; (2) it comprehensively compares the four regionalisation methods across the continental Australia; (3) it demonstrates that using the gridded integrated similarity approach outperforms using the spatial proximity in data sparse inland and central Australia, which is particularly important since most Australian catchments locate along coastal regions, but the inland and central Australia has very sparse gauges. We acknowledge the criticism that poor language was used. We will try our best to improve its readability in the next version if the editor provides us a revision opportunity. (End of response)

II. Specific comments Page 1, abstract needs to be restructured: recapitulating the intention of the study, the novelty of the analysis and how it could be useful; key points about how these could be supported by the main findings. Page 2, introduction needs to be fulfilled with deep thinking on status quo, and what this study will bring or add on; with more insightful discussions on literature research. Page 4, line 1 and 2, why these 3 examples are listed here? Any particular reasons to select these from the long list in Table 1? Add. more discussions. Page 4, line 5, "may produce different conclusions between studies", here needs more details. Page 4, line 8, what "descriptors"? please elaborate. Page 4, line 10, not enough support to come to this conclusion. Page 4, line 12, it seems not true, there are many other studies, e.g. Oudin et al. 2008 Page 4, line $15-28$, not sufficient argument why the authors chose these four methods, two models, and what's the value to compare the methods, models, and why it's applied to those catchments in Australia? Actually after reading the whole paper, still no clear idea on what's the purpose of this study and what's the benefit?

Re: Page 1, yes we would like to restructure abstract to articulate the intention and novelty of this study, and then show the key results followed by main findings. Page 2 , yes we would like to have deeper thinking on status quo. Page 4 , we need to clarify that these are just examples and more examples are summarised in Table 1. We will have more discussion on Table 1. We would like to show more details on

Printer-friendly version

Discussion paper 
the statement "may produce different conclusion between studies". Page 4, line 8, "descriptors" should be replaced by "characteristics". Page 4, line 10, we would soften our language for doing large-scale regionalisation studies Page 4, line 12, Oudin et al. 2008 only used 913 France catchments, noting to do with continental daily runoff prediction study. The reviewer states "it seems that it is not true...", he or she DID NOT provide any reference to prove what our statement is not correct. Page 4, line 15-28, it is really astonished to see this kind of comment ".. chose four methods, two models. ..". Choose of two models can make our conclusion more robust. Are there any wrong with this? Comparing four regionalisation methods can have a comprehensive evaluation of the various regionalisation methods. Are there any wrong with that? We will try to articulate the manuscript objectives. (End of response)

Page 5 and 6, Data section needs more details and to be addressed in a more meaningful way and in a logic structure. Just list a few example here, more can be found in text and need to be revised. Page 5, line 17, what's the meaning of "revised" rainfallrunoff models? Page 5, line 20, please add more details for the daily data of 605 catchments "collated by zhang et al. 2013" Page 5, line 21, please state why "data from 1975 to 2012 are used in this study". Page 5, line 26, \& page6, any reference or source for "MODIS", "International Geosphere-Biosphere Programme"? There are many other similar things need to quote reference properly. Page 6 , line 1-10, it's not clearly stated where and how data was obtained, produced, or processed. Please either cite original data sources, or data processing method, or quote reference properly, and in a meaningful, easy-understandable way..

Re:. Page 5, line 17, what's the meaning of "revised" rainfallrunoff models? We should clarify here that the detail for the revised rainfall-runoff model is introduced in section 3.1 Page 5, line 20, please add more details for the daily data of 605 catchments. Yes, we would put more details on the daily runoff data of 605 catchments. why "data from 1975 to 2012 are used in this study". This data set comes from the national water accounting project which collocated daily streamflow data for 1975 to 2012, which

Printer-friendly version

Discussion paper 
cover long-period of time, and different climate conditions, being good enough for any regionalisation studies.

Modis issue. We will show a reference for the MODIS dataset. Yes, we will show more details on IGBP land cover types

We will introduce more clearly on data source, data processing processing method and quote reference appropriately in next round of revision. (End of response)

Section 3-6, Poor language, poor structure, lack of detailed description, lack of meaningful discussion, no adequate justification through all these sections. Considerable modification will be required, and suggest having someone review the article before submission.

Re: We accept the criticism of this review. We will try our best to more accurately summarise our results, to have more meaningful and thoughtful discussion, and will ask a peer to review it again

This reviewer's thoughtful thinking will make us to present our manuscript more accurately. We will follow his/her suggestions for rephrase text and manuscript structure. . (End of response)

Why these objective functions were selected? How to do the model calibration and evaluation? What are the conditions to relate donor and ungauged catchments? Why select these five properties to define catchment similarity? Please embed more discussions and justifications in these sections, to lead to meaningful conclusion.

Re: The objective function using NSEsqrt focuses on not only high daily flow but low daily flow as well; the bias is the evaluation of accuracy for mean annual runoff. This is the objective function widely used for rainfall-runoff modelling.

Printer-friendly version

Model calibration. A global model calibration method, the genetic algorithm, was used for model calibration. Model evaluation. Regionalisation evaluation. We should clarify this, should not we? Conditions of donor and ungauged catchments. we should clarify 
that all the catchments selected are met several criteria: (1) unregulated; (2) without subject to noticeable urbanisation; (3) streamflow data length more than 10 years; (4) catchment area less than $5000 \mathrm{~km} 2$.

The five properties. We have had a section to discuss this. In section 5.5, the text states that "5.5 Practical ways for selecting predictors to build the gridded IS approach. It is necessary to select the IS predictors that are easily available and representative for macro-scale runoff prediction studies. This study chooses five predictors to build the similarity indices. Among them, the aridity index reflects climate wetness or dryness; the fraction of forest ratio reflects the vegetation condition; the mean annual air temperature represents both climate and elevation; and the two rainfall indices represent rainfall seasonality and the standard deviation of daily rainfall. These predictors are relatively easily obtained and representative and are believed to be sufficient for continental Australia or other warm regions. It is possible that the current selected predictors are not enough for the high latitude northern hemisphere or high elevation regions where snow melt is often a major contributor to runoff, and therefore, extra predictors, such as permanent snow cover, snowfall percentage, and days with a mean daily temperature less than $0^{\circ} \mathrm{C}$, should be included as well.". We should put more argument for the choice, such as we did correlation analysis first and picked up the five with good correlations and they are representative.

Five donor catchments, we should more clearly explain that. We did this based on numerous donor catchment number sensitivity analysis, as indicated by Zhang and Chiew, (2009) and Oudin et al. (2008). (End of response)

III. Technical comments All the equations should be in a consistent format, and also for the paragraph after the equation which explains all the parameters in equation. Many sections are too short to be a section, e.g. 3.5, 3.6, some has just one sentence. In the 1st paragraph of section 4, all figures are mentioned together, this is not a good way to state the results. Please revise and prefer to talk about them one by one, with discussion.

Printer-friendly version

Discussion paper 
Re: We accept the criticism, and are preparing to improve the manuscript as suggested by this reviewer.

In summary, we thank dearly to this reviewer for his/her thoughtful thinking and detailed points which can easily help us to improve our MS during next round of revision. Overall the critical points do not influence the key conclusions drawn from this study. The merit of this study should be more clearly communicated and articulated. We are preparing to do so. (End of response)

Interactive comment on Hydrol. Earth Syst. Sci. Discuss., doi:10.5194/hess-2016-464, 2016. 